\title{
Large-Vessel Vasculitides: Takayasu Arteritis and Giant Cell Arteritis
}

\author{
Giulio Cavalli, Giacomo De Luca, \\ and Lorenzo Dagna
}

\subsection{Takayasu Arteritis}

\subsubsection{Clinical Features}

Takayasu arteritis (TA) is a chronic, large-vessel granulomatous vasculitis of unknown etiology, which primarily affects the aorta and its primary branches ("aortic arch syndrome") [1]. TA chiefly affects young women: patients are female in $80-90 \%$ of cases, and the age of onset is typically before 40 years, with a mean age at diagnosis between 25 and 30 years. It has a worldwide distribution, with peak prevalence in Asian countries $(0.004 \%$ in Japan versus 4.7 cases per million in the UK).

The etiology of TA is largely obscure [2]. A genetic predisposition for the immune-mediated process is postulated, but disease pathogenesis and natural history are poorly understood. The histologic hallmark of TA is granuloma formation and transmural infiltration with inflammatory cells, eventually leading to a marked thickening of the walls of affected arteries. Despite remarkable variability in disease expression, the initial vascular lesions frequently occur in the left middle or proximal subclavian artery (Fig. 35.1). As the disease progresses, the left common

G. Cavalli · G. De Luca $\cdot$ L. Dagna $(\bowtie)$

Unit of Immunology, Rheumatology, Allergy and Rare Diseases, San Raffaele Hospital and University, Milan, Italy

e-mail: dagna.lorenzo@hsr.it carotid, vertebral, brachiocephalic, right middle or proximal subclavian artery, right carotid, vertebral arteries, and aorta may also be affected. The abdominal aorta and pulmonary arteries are involved in approximately $50 \%$ of patients. Vascular inflammation may be localized to a portion of the thoracic or abdominal aorta and branches or may extensively involve the entire vessels. The inflammatory process within the

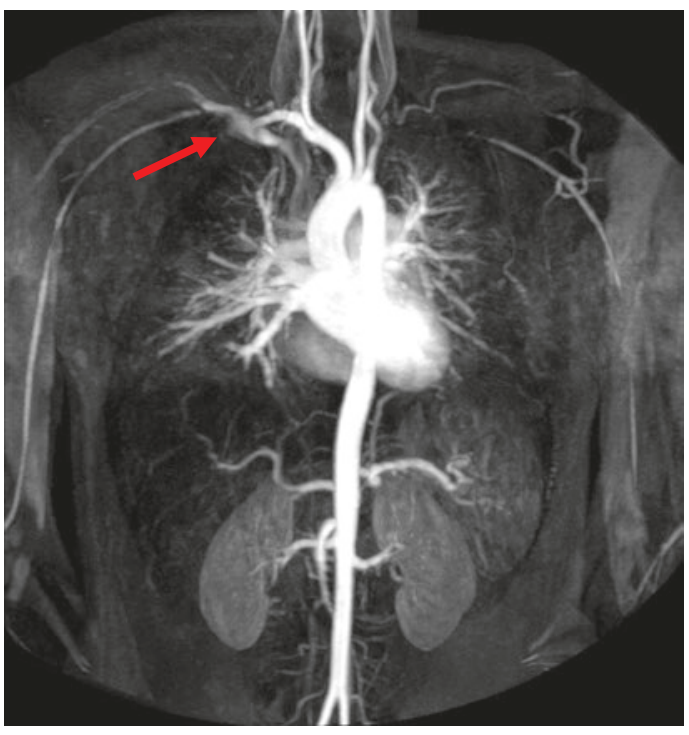

Fig. 35.1 CMR angiography in large-vessel vasculitides. Contrast-enhanced magnetic resonance angiography coronal plane showing diffuse luminal irregularities without significant stenosis and marked right subclavian wall thickening up to $25 \mathrm{~mm}$ (arrowheads) with intense contrast enhancement 
vessel can lead to narrowing, occlusion, or dilation of involved portions of the arteries, which cause a wide variety of symptoms.

Clinical presentation is highly variable and varies from nonspecific constitutional symptoms without clinical or imaging evidence of arterial occlusive disease (pre-pulseless stage) to symptoms attributable to arterial stenosis, aneurysm, and occlusion (pulseless stage) [3]. At time of disease onset, the clinical picture tends to be subacute, for example, with nonspecific constitutional symptoms (i.e., fatigue, malaise, night sweats, weight loss, fever) and arthralgia. This elusive clinical picture often causes a remarkable delay in diagnosis, ranging from months to years, during which vascular disease likely progresses. Although vascular involvement is often asymptomatic at presentation, it later becomes clinically apparent as dilation, narrowing, or occlusion of arteries develops, resulting in a broad spectrum of vascular symptoms including limb cyanosis and claudication, lightheadedness or other symptoms of reduced blood flow, vascular bruits, arterial pain and tenderness (i.e., carotidynia), and neurovascular hypertension. The pulseless phase is characterized by vascular insufficiency manifesting as arm or leg claudication and neurological symptoms due to decreased blood flow to the brain. Rare but alarming complications of TA include ocular involvement in form of various degrees of visual field defects, coronary artery aneurysms, as well as rupture of aneurysms in other sites [4].

\subsubsection{Diagnosis}

TA should be suspected in a young patient, chiefly woman, who has constitutional symptoms, hypertension, diminished or absent pulses, and/or arterial bruits. At physical examination, accurate measurements of blood pressure and palpation of pulses may reveal asymmetric or discordant findings. Absent or weak peripheral pulses are most common at the level of the radial arteries and led to the usual definition of "pulseless disease." Vascular bruits or a murmur consistent with aortic valve insufficiency are common.
There are no gold standard imaging or laboratory tests with adequate sensitivity or specificity for the diagnosis of TA [5]. Testing for inflammatory markers such as the erythrocyte sedimentation rate (ESR) and the acute-phase reactant $\mathrm{C}$-reactive protein (CRP) indicates the presence of a systemic inflammatory process; however, normal values may occasionally be observed and should not unequivocally rule out a diagnosis of TA. Anemia often accompanies the earliest inflammatory stages of the disease. In most cases, the diagnosis is based on suggestive clinical features and specific imaging findings of the aorta and/or its branches. In these regards, TA is sometimes incidentally suspected upon findings of arterial disease at imaging obtained for other clinical indications or revealed when vasculitis is observed on histologic examination of surgically removed arteries.

Imaging studies of the arterial tree are essential for ascertaining the diagnosis of TA and for determining the extent and severity of vascular involvement [6].

Preferred techniques are magnetic resonance angiography (MRA) and computed tomography angiography (CTA), which allow for evaluation of the arterial lumen. MRA use circumvents the risks of exposure to radiation and iodinated contrast of CTA; since periodic re-evaluations can be anticipated, MRA is the preferred technique. Imaging of the vasculature of the chest, abdomen, head and neck, or other areas by MRA or CTA demonstrates smoothly tapered luminal narrowing or occlusion, which may sometimes be accompanied by circumferential thickening of the vessel wall. The characteristic finding on contrast angiography is the presence of "skip lesions," where stenosis or aneurysms alternate with normal vessels. FDG-PET can help in diagnosing an active inflammation, providing additional information apart from vessel anatomy and patency, and thus resulting particularly useful in monitoring disease activity during follow-up [7] (Fig. 35.2).

Conditions that should be considered in the differential diagnosis of TA include other diseases which can feature large-vessel vasculitis or aortitis, such as giant cell (temporal) arteritis, 


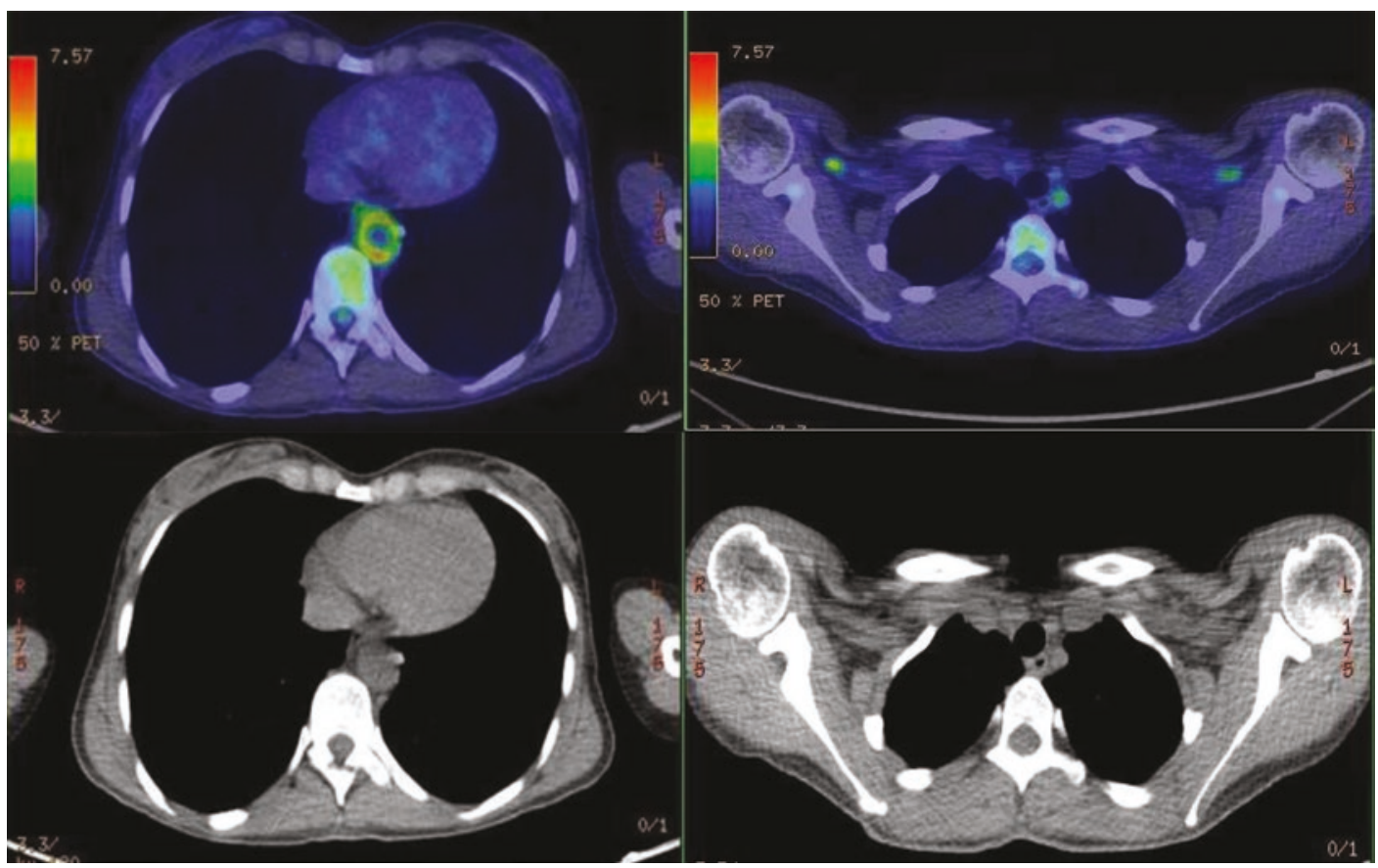

Fig. 35.2 CT/F-FDG-PET in large-vessel vasculitides (Takayasu arteritis). CT/PET showing FDG uptake in the thoracic aorta and in both subclavian arteries

Cogan's syndrome, Behçet's syndrome, relapsing polychondritis, infectious aortitis, atherosclerosis, fibromuscular dysplasia, and genetically determined causes of aneurysms such as EhlersDanlos syndrome, IgG4-related disease, and spondyloarthropathies.

\subsubsection{Treatment}

TA is a chronic, relapsing, and progressive disease requiring long-term immunosuppression [8].

Glucocorticoids The cornerstone of therapy for TA is glucocorticoids. High-dose glucocorticoids effectively control systemic inflammation and related clinical manifestations (fever, arthritis or arthralgia, normochromic anemia, and elevated acute-phase reactants) in the majority of cases and can arrest or fully revert the progression of vascular inflammation and damage of TA. In early cases, arterial stenosis may reverse, and ischemic symptoms may improve remarkably upon corticosteroid treatment. On the other and, in later disease stages, fibrous damage may develop and be poorly responsive to treatment.

A daily dose of 45-60 $\mathrm{mg}$ of prednisone $(0.5-1 \mathrm{mg} / \mathrm{Kg})$ is typically considered an adequate starting dosing for adults of average size. A single morning is convenient and preferred, as divided doses are associated with an increased number of adverse reactions.

Gradual tapering when symptoms and laboratory analyses related to the inflammatory process have improved follows this loading steroid dose. Long-term, low-dose prednisone therapy may be necessary to prevent progression of arterial stenoses. Glucocorticoids can be discontinued if the disease enters prolonged remission, but the dose should be increased if flares occur. Supplementation with calcium and vitamin D, as well as prophylactic use of an oral bisphosphonate, is recommended to prevent glucocorticoid-related osteoporosis, since duration and cumulative dose of treatment cannot be predicted at time of initiation. 
Evaluation of Disease Activity and Response to Treatment Assessing residual disease activity and response to treatment is a challenging task, given the lack of specific laboratory tests or validated tools for assessing disease activity. Clinical evaluations include monitoring of constitutional or vascular symptoms and levels of acute-phase reactants (ESR and CRP). Efforts aimed at developing a quantitative clinical assessment instrument are undergoing. The Indian Takayasu Clinical Activity Score (ITAS 2010) and a composite ITAS-A, which also includes acute-phase reactants (ESR or CRP), are currently used for research purposes and provide a quantitative score of clinical disease activity for patient monitoring [9].

Imaging techniques provide some aid in evaluating disease activity and response to treatment. CTA, MRA, and FDG-PET can be used.

\section{Glucocorticoid-Resistant}

Disease

Approximately, one-half of all TA patients exhibit chronically active disease and vascular inflammation refractory to treatment with glucocorticoids. These patients normally benefit from the adjunctive use of immunosuppressant medications. Drugs used in the management of TA include methotrexate, azathioprine, leflunomide, and mycophenolate mofetil [8]. In small openlabel studies, the use of these medications in association with glucocorticoids was generally associated with a significant reduction in disease activity scores, acute-phase reactants, and glucocorticoid use. More sparse evidence and limited experience indicate that mycophenolate mofetil and cyclophosphamide may have some value as steroid-sparing agents in patients with TA refractory to conventional therapy. There is currently no evidence to clearly privilege any of these drugs over others. Comparative randomized trials can hardly be conceived and populated, given the small size of patient in individual centers.

Pharmacologic blockade of pro-inflammatory cytokines with monoclonal antibodies represents a promising option for difficult-to-treat patients. Experience with antitumor necrosis factor (TNF)- alpha agents infliximab or etanercept in TA is limited, but available evidence point at a role for these agents in the management of difficult-totreat patients. Emerging evidence indicates that blockade of interleukin (IL)-6 with tocilizumab may also be effective in TA; of note, this drug exhibited remarkable efficacy in a larger number of patients with giant cell arteritis [10].

Revascularization Despite medical treatment, irreversible arterial stenosis or significant ischemic symptoms may develop in late cases and necessitate treatment with percutaneous transluminal angioplasty, bypass grafts, or other surgeries. In general, surgical treatments yield better outcomes than endovascular interventions. In a long-term follow-up study of 106 Japanese patients who required surgical revascularization, survival to hospital discharge was $89 \%$ and overall survival $73 \%$. Of note, aneurysms at sites of anastomosis eventually developed in $13.8 \%$ of long-term survivors, requiring lifelong surveillance for the development of this complication.

Percutaneous angioplasty and stenting are burdened with frequent postoperative complications, the most common of which is restenosis, occurring in $77 \%$ of angioplasty procedures, as opposed to $34 \%$ of surgical bypasses. Nevertheless, angioplasty may be preferred in the event of lesions amenable to catheter-based dilation. Stenosis or occlusions affecting sizeable tracts of an artery, as well as heavy scarring of vessels, may reduce both the feasibility and the success rate of percutaneous revascularization [11].

Of note, a state of active inflammation prior to or after revascularization is associated with poor outcomes, regardless of procedure modality. Complications include graft detachment or restenosis, new arterial lesions, and development of progressive symptomatic disease. It is thus crucial that disease activity is controlled with adequate immunosuppressive treatment prior to or - in case of urgent revascularization - at least following a procedure [12].

Aortic Valve Surgery Progressive aortic regurgitation (AR) may develop when the ascending 
aorta is involved. This complication may require surgical valve replacement or repair. The state of inflammation and fragility of affected walls make this surgery more challenging than usual. In a large series of 90 patients (63 undergoing aortic valve replacement and 27 undergoing composite graft repair), in-hospital mortality was $5.5 \%$, and 15 -year survival was $76 \%$; the long-term outcome after surgery seemed comparable between these two approaches [13].

\subsubsection{Prognosis}

The development of TA marks the onset of a severe, chronic disease in the most productive years of life. Vascular involvement tends to be progressive, and disease activity may vary over time leading to occasional exacerbations of the inflammatory process; however, spontaneous remissions also rarely occur as the disease may eventually burn out.

The short-term prognosis of TA is generally favorable, with several follow-up studies reporting a 5-year survival rate of $80-90 \%$. The overall mortality has declined over time such that the 15-year survival rate has increased from $82.9 \%$ for patients diagnosed between 1957 and 1975 to $96.5 \%$ for those diagnosed from 1976 to 1990 . However, the 15-year survival rate may drop to $40-70 \%$ in those TA patients with a progressive course and major complications, including retinopathy, hypertension, aortic regurgitation, and aneurysms. In these subsets of patients, more aggressive medical and/ or surgical therapy is warranted to prevent an unfavorable outcome [14].

\subsection{Giant Cell Arteritis}

\subsubsection{Clinical Features}

Giant cell arteritis (GCA), or Horton's disease, is a systemic vasculitis of unknown etiology. GCA is historically classified as a large-vessel vasculitis chiefly involving the cranial arteries, typically the temporal artery ("temporal arteritis")
[15]. However, GCA exhibits a larger disease spectrum also including idiopathic aortitis and small-medium vessel involvement. The disease, indeed, may also involve the aorta and carotid, subclavian, iliac arteries and the superficial temporal, ophthalmic, occipital, vertebral, posterior ciliary, and proximal vertebral arteries. Given this lesion distribution, GCA may cause a variety of systemic, neurologic, and ophthalmologic complications.

The incidence of GCA peaks between age 70 and 80 years, and the disease occurs with greater frequency in females ((female-to-male ratio 3.7:1) and in Nordic countries. GCA virtually never occurs before the age of 50, making GCA the most common systemic vasculitis in adults with a reported incidence of $0.5-27$ cases per 100,000 people aged 50 years or older [16, 17].

GCA is often associated with polymyalgia rheumatica (PMR), an inflammatory condition characterized by inflammatory pain in the shoulder and hip girdles. PMR occurs in about $50 \%$ of patients with GCA, and GCA may develop in about 15\% of patients with PMR [18]. Musculoskeletal symptoms in addition to those of PMR can be observed in a minority of patients.

The onset of GCA may be either abrupt or insidious, and clinical presentation is highly variable from mild constitutional symptoms to complete visual loss [19]. Prodromal constitutional symptoms such as anorexia, fatigue, low-grade fever, malaise, myalgia, night sweats, weight loss, and nonproductive cough may herald the disease. A common presentation is the onset of temporal or occipital headache in a patient without a history of headaches or a new, unusual complaint in a patient with a history of chronic headache $[18,20]$.

Jaw claudication is also common and highly predictive, and it is reported as fatigue or discomfort of the jaw muscles upon prolonged chewing or speaking; it is due to ischemia of the maxillary artery supplying the masseter muscles. Involvement of other branches of the external carotid artery may result in many other extracranial GCA symptoms, including maxillary and dental pain, facial swelling, throat pain, and tongue pain. 
Visual symptoms are a common, feared manifestation of GCA [21]. Transient monocular (and, rarely, binocular) impairment of vision can be an early manifestation of GCA. Patients typically note an abrupt partial field defect or temporary curtain effect in the field of vision of one eye. Transient episodes of blurred vision are usually reversible, but sudden and painless irreversible loss of vision may occur if treatment is not started promptly [22]. Permanent loss of vision in GCA results from anterior ischemic optic neuropathy ( $80 \%$ of cases), central or branch retinal arterial occlusion, posterior ischemic optic neuropathy, or, rarely, posterior cerebral ischemia [23]. Loss of vision may be partial or complete and unilateral or bilateral. Once established, visual loss is rarely reversible. Before the advent of glucocorticoid treatment, the prevalence of visual complications was high. Increasing awareness by physicians of the symptoms of GCA and advances in diagnostic techniques over the past 20 years have also contributed to a substantial decline in the frequency of permanent visual loss.

GCA can also affect the central nervous system [24]: involvement of the vertebral arteries can cause vertigo, ataxia, dysarthria, homonymous hemianopsia, or bilateral cortical blindness; bilateral vertebral artery involvement can cause rapidly progressive brain stem and/or cerebellar neurologic deficits. Stroke is uncommon but may derive from involvement of vertebrobasilar or internal carotid arteries.

GCA can also involve extracranial arteries, chiefly the aorta and its major proximal branches, especially in the upper extremities: the subclavian arteries distal to the emergence of the vertebral arteries and extend through the axillary arteries to the proximal brachial arteries. Arterial bruits, diminished or absent pulses, arm claudication, and cold intolerance are possible complaints. Patients with these peripheral presentations of GCA are usually younger at diagnosis and have fewer cranial symptoms. Complications include aortic aneurysms and dissection, particularly affecting the thoracic aorta, as well as stenosis, occlusion, and ectasia of large arteries. While these complications occur in a minority of GCA patients, subclinical or clinical aortitis is commonly detected at imaging $[25,26]$.

As incidence of aortic aneurysms in GCA increases over time, especially in male smokers, ongoing clinical vigilance is warranted. Imaging modalities for diagnosing large-vessel involvement in GCA include color Doppler ultrasonography, CT or CT with angiography (CTA), MRI or MR angiography (MRA), and FDG-PET or PET with CT.

\subsubsection{Diagnosis}

The diagnosis of GCA should be considered in a patient over the age of 50 years with new headaches, abrupt onset of visual disturbances (especially transient monocular visual loss), jaw claudication, unexplained constitutional symptoms, and inflammatory manifestations including fever, anemia, elevated ESR, and/or CRP. A current or prior diagnosis of polymyalgia rheumatica strengthens the predictive value of any of these findings.

At physical examination, the temporal or other cranial arteries can be tender or thickened. Bruits may be heard on auscultation of the carotid or supraclavicular areas: over the axillary, brachial, or femoral arteries. Murmurs over the abdominal aorta and of aortic regurgitation may reveal the development of an aortic aneurysm.

The laboratory hallmark of GCA is an elevation in ESR and CRP levels. The ESR usually exceeds $50 \mathrm{~mm} / \mathrm{h}$ but may be normal in $7-20 \%$ of patients with GCA; thus, a normal ESR does not rule out GCA. CRP is typically elevated. Combined assessment of ESR and CRP provides a remarkable sensitivity for the diagnosis of GCA [27, 28].

The definite diagnosis of GCA is based on histopathology on temporal artery biopsy. Histologic confirmation should be obtained almost without exception in patients with clinically suspected GCA, since glucocorticoid treatment is prolonged and often complicated. A positive temporal artery biopsy has $100 \%$ specificity; however, sensitivity is relatively low $(15-87 \%)$ due to a 
"skip lesion" pattern of disease involvement, with normal intervening segments [29]. For this reason, large $(5 \mathrm{~cm})$ and occasionally bilateral specimens should be obtained. In the setting of a high diagnostic suspicion for GCA, scheduling of the biopsy should not interfere with initiation of glucocorticoid therapy due to concerns for possible catastrophic sight loss [30].

Color Doppler ultrasonography of the temporal artery has emerged as a promising alternative or complement to temporal artery biopsy, with a specificity of $80-100 \%$ when a dark halo surrounds the vessel ("halo sign") [31]. This key diagnostic feature is believed to represent vessel wall edema.

The following are classification criteria for GCA issued by the American College of Rheumatology in 1990 [32]:

- Age 50 years or older

- New-onset localized headache or localized head pain

- Temporal artery tenderness to palpation or decreased pulsation

- ESR of $50 \mathrm{~mm} / \mathrm{h}$ or higher

- Positive arterial biopsy results (vasculitis characterized by mononuclear infiltration or granulomatous inflammation, usually with multinucleated giant cells)

The presence of three or more criteria yields a diagnostic sensitivity of $93.5 \%$ and specificity of $91.2 \%$.

\subsubsection{Treatment}

Immunosuppressive Treatment The mainstay of treatment for GCA is high-dose corticosteroids. For patients in whom the diagnostic suspicion of GCA is high, especially those with recent vascular complications such as visual loss, therapy should be started promptly, even before the diagnosis is confirmed. A temporal artery biopsy should be obtained as soon as possible, but treatment should not be delayed while awaiting biopsy.
For GCA not complicated by ischemic organ damage (i.e., visual loss), treatment is started at a single glucocorticoid dose equivalent to 40-60 mg of prednisone [33]. However, in the setting of visual loss, damage may be irreversible if treatment is delayed beyond $48 \mathrm{~h}$, and intravenous pulse methylprednisolone (1000 mg intravenously each day for 3 days, followed by oral therapy with $1 \mathrm{mg} / \mathrm{kg}$ per day) is recommended. High-dose prednisone should be continued at the starting dose for 2-4 weeks, followed by tapering by approximately $10 \%$ of the total daily dose each 1 or 2 weeks. After achieving a daily dose of $10 \mathrm{mg}$, the prednisone taper should be slowed substantially, so that patients remain on progressively lower doses for 9 to 12 months. The risk of disease flare increases as the daily prednisone dose declines. Supplementation with calcium, vitamin D, bisphosphonate therapy, antimicrobial prophylaxis, and initiation of a proton pump inhibitor or histamine H2-receptor antagonist should be considered. Low-dose aspirin in association to corticosteroids decreases the risk of cranial ischemic complications (visual loss, transient ischemic attacks, or stroke).

Oral corticosteroid therapy is required for months to years with careful follow-up and periodic laboratory evaluations with ESR and CRP: recurrence of inflammation is common in GCA and necessitates an escalation of corticosteroid dose and/or a second-line therapy. Immunomodulatory therapy may be considered in patients experiencing relapsing inflammation despite high doses of corticosteroids or those with corticosteroid-induced complications. Emerging evidence for adjunctive suitable steroid-sparing agents such as methotrexate and the anti-IL-6 tocilizumab is encouraging and may lead to a more mainstream role for these therapies among patients with GCA [34].

Surgery Despite medical treatment, irreversible arterial stenosis or significant ischemic symptoms may develop in late stages in rare cases and necessitate treatment with percutaneous transluminal angioplasty, bypass grafts, or other surgeries. A surgical approach is rarely needed in GCA with 
extracranial involvement. Moreover, when endovascular revascularization procedures and open surgical treatment for aneurysms and dissections are necessary, results are not always favorable and relapses are common. Given the elderly population of patients with GCA, comorbidities often limit surgical interventions [25].

\subsubsection{Prognosis}

The prognosis of untreated GCA is extremely poor: serious disability may occur from blindness, or death may result from myocardial infarction, stroke, or dissecting aortic aneurysm. However, in most treated patients, GCA tends to have a self-limited course over several months to several years and may not adversely affect overall survival. Evidence is emerging that overall mortality in GCA patients is at best slightly higher than expected in relation to general population mortality data, but GCA is associated with an increase in morbidity and mortality specifically related to aortic aneurysm or other cardiovascular causes [35].

Glucocorticoid therapy can eventually be reduced and discontinued in the majority of patients. The burden of morbidity from steroid therapy is significant and includes diabetes mellitus, hypertension, osteoporosis, steroid myopathy, steroid psychosis, and immunosuppression-related infections.

\section{References}

1. Arend WP, et al. The American College of Rheumatology 1990 criteria for the classification of Takayasu arteritis. Arthritis Rheum. 1990;33(8):1129.

2. Weyand CM, Goronzy JJ. Medium- and large-vessel vasculitis. NEJM. 2003;349(2):160.

3. Mason JC. Takayasu arteritis--advances in diagnosis and management. Nat Rev Rheumatol. 2010;6(7):406-15.

4. Serra R, et al. Updates in pathophysiology, diagnosis and management of Takayasu arteritis. Ann Vasc Surg. 2016;35:210-25.

5. Kim E, et al. Takayasu arteritis: challenges in diagnosis and management. Heart. 2017;104(7):558-65.

6. Alibaz-Oner F, et al. Update on Takayasu's arteritis. Presse Med. 2015;44:e259-65.
7. Barra L, et al. Imaging modalities for the diagnosis and disease activity assessment of Takayasu's arteritis: a systematic review and meta-analysis. Autoimmun Rev. 2018;17(2):175-87.

8. Keser $\mathrm{G}$, et al. What is new in management of Takayasu arteritis? Presse Med. 2017;46:e229-35.

9. Misra R, et al. Development and initial validation of the Indian Takayasu Clinical Activity Score (ITAS2010). Rheumatology (Oxford). 2013;52:1795-801.

10. Abisror N, et al. Tocilizumab in refractory Takayasu arteritis: a case series and updated literature review. Autoimmun Rev. 2013;12:1143-9.

11. Kazibudzki M, et al. New endovascular techniques for treatment of life-threatening Takayasu arteritis. Adv Interv Cardiol. 2016;12:171-4.

12. Saadoun D, et al. Retrospective analysis of surgery versus endovascular intervention in Takayasu arteritis: a multicenter experience. Circulation. 2012;125:813-9.

13. Matsuura K, et al. Surgical treatment of aortic regurgitation due to Takayasu arteritis: long-term morbidity and mortality. Circulation. 2005;112(24):3707.

14. Schmidt J, et al. Diagnostic features, treatment, and outcomes of Takayasu arteritis in a US cohort of 126 patients. Mayo Clin Proc. 2013;88(8):822.

15. Jennette JC, et al. 2012 revised international Chapel Hill consensus conference nomenclature of vasculitides. Arthritis Rheum. 2013;65(1):1-11.

16. Goodwin JS. Progress in gerontology: polymyalgia rheumatica and temporal arteritis. J Am Geriatr Soc. 1992;40(5):515-25.

17. Gonzalez-Gay MA, et al. Epidemiology of giant cell arteritis and polymyalgia rheumatica. Arthritis Rheum. 2009;61(10):1454.

18. Gonzalez-Gay MA, et al. Giant cell arteritis: disease patterns of clinical presentation in a series of 240 patients. Medicine (Baltimore). 2005;84(5):269.

19. Buttgereit F, et al. Polymyalgia rheumatica and giant cell arteritis: a systematic review. JAMA. 2016;315(22):2442-58.

20. Myklebust G, Gran JT. A prospective study of 287 patients with polymyalgia rheumatica and temporal arteritis: clinical and laboratory manifestations at onset of disease and at the time of diagnosis. $\mathrm{Br} \mathrm{J}$ Rheumatol. 1996;35(11):1161-8.

21. González-Gay MA, et al. Visual manifestations of giant cell arteritis. Trends and clinical spectrum in 161 patients. Medicine (Baltimore). 2000;79(5): 283-92.

22. Salvarani C, et al. Risk factors for visual loss in an Italian population-based cohort of patients with giant cell arteritis. Arthritis Rheum. 2005;53(2):293-7.

23. Soriano A, et al. Visual loss and other cranial ischaemic complications in giant cell arteritis. Nat Rev Rheumatol. 2017;13:476-84.

24. Gonzalez-Gay MA, et al. Strokes at time of disease diagnosis in a series of 287 patients with biopsyproven giant cell arteritis. Medicine (Baltimore). 2009;88(4):227-35. 
25. Lensen $\mathrm{KD}$, et al. Extracranial giant cell arteritis. Neth J Med. 2016;74(5):182-92.

26. Nuenninghoff DM, et al. Incidence and predictors of large-artery complication (aortic aneurysm, aortic dissection, and/or large-artery stenosis) in patients with giant cell arteritis: a population-based study over 50 years. Arthritis Rheum. 2003;48:3522-31.

27. Weyand CM, Goronzy JJ. Clinical practice: giant-cell arteritis and polymyalgia rheumatica. $\mathrm{N}$ Engl $\mathrm{J}$ Med. 2014;371(1):50-7.

28. Gonzalez-Gay MA, et al. Giant cell arteritis: laboratory tests at the time of diagnosis in a series of 240 patients. Medicine (Baltimore). 2005;84(5):277.

29. Niederkohr RD, Levin LA. A Bayesian analysis of the true sensitivity of a temporal artery biopsy. Invest Ophthalmol Vis Sci. 2007;48(2):675-80.

30. Jakobsson K, et al. The effect of clinical features and glucocorticoids on biopsy findings in giant cell arteritis. BMC Musculoskelet Disord. 2016;17(1):363.

31. Dejaco C, et al. EULAR recommendations for the use of imaging in large vessel vasculitis in clinical practice. Ann Rheum Dis. 2018;77:636-43.

32. Hunder GG, et al. The American College of Rheumatology 1990 criteria for the classification of giant cell arteritis. Arthritis Rheum. 1990;33: 1122-8.

33. Dasgupta B, et al. BSR and BHPR guidelines for the management of giant cell arteritis. Rheumatology (Oxford). 2010;49:1594-7.

34. Dejaco C, et al. Giant cell arteritis and polymyalgia rheumatica: current challenges and opportunities. Nat Rev Rheumatol. 2017;13:578-92.

35. Hill CL, et al. Risk of mortality in patients with giant cell arteritis: a systematic review and meta-analysis. Semin Arthritis Rheum. 2017;46(4):513-9. 